\title{
CROSS-PLANE RESONANCE: A MECHANISM FOR VERY LARGE AMPLITUDE HALO FORMATION
}

\author{
J. Qiang*, LANL, Los Alamos, NM 87545, USA \\ I. Hofmann, GSI, 64291 Darmstadt, Germany \\ R. D. Ryne, LBL, Berkeley, CA 94720, USA
}

\begin{abstract}
In this paper we report on the possibility of very large amplitude halo formation due to a cross-plane resonance, based on a core/test-particle model. Previous analyses of halo formation in mismatched beams (which did not involve a coupling of the phase planes) have found that particles may be driven to amplitudes which are limited to typically three times the edge radius by a $2: 1$ parametric resonance. In the present paper, we systematically study the transverse halo as functions of the longitudinalto-transverse focusing strength ratio and the longitudinalto-transverse emittance ratio. We find that, in cases where the longitudinal focusing exceeds the transverse focusing, the parameters may lead to a new type of resonance, a coupled-plane resonance, where the transverse amplitude can be, in principle, unlimited. In this regime (in contrast to the usual in-plane resonance), the halo amplitude increases with a reduction of the space charge. This may have potential relevance to the future superconducting linac design where the longitudinal to transverse focusing ratio could be greater than one.
\end{abstract}

\section{INTRODUCTION}

The particle-core model has been widely used to study halo formation in high intensity linacs. In this model, the beam core is described by rms envelope equations, and the halo particles are modeled using test particles that are subject to the external focusing force and the timedependent, nonlinear space-charge force associated with the core. The test particles do not affect the motion of the core. The oscillation frequency of test particles is amplitude-dependent. This results in some particles being driven to large amplitudes through a parametric resonance with the core [1]. Previous studies with the longitudinalto-transverse focusing ratio less than one showed that the halo amplitude was about two to three times the matched beam size $[2,3,4,5,6]$. With the longitudinal-to-transverse focusing strength ratio greater than one, this may significantly increase the transverse halo amplitude through a cross-plane resonance as described below.

\footnotetext{
*jiqiang@lanl.gov
}

\section{TRANSVERSE AND LONGITUDINAL HALO FORMATION}

The calculations that follow are based on a threedimensional particle-core model [7]. The physical parameters used in our study are given in Table 1 . They are similar to those of the present spallation neutron source superconduting linac design [8]. We first study halo formation

Table 1: Physical parameters

\begin{tabular}{|l|c|}
\hline Bunch Current $(\mathrm{A})$ & 0.112 \\
\hline Proton Energy (MeV) & 199.29 \\
Synchronous Phase (degrees) & -30 \\
rf Frequency (MHz) & 805 \\
Trans. Focusing Gradient $(\mathrm{rad} / \mathrm{m})$ & 0.228 \\
Long. Focusing Gradient $(\mathrm{rad} / \mathrm{m})$ & 0.242 \\
Trans. Unnorm. Emittance $(\pi-\mathrm{mm}-\mathrm{mrad})$ & 0.4 \\
Long. Unnorm. Emittance $(\pi-\mathrm{mm}-\mathrm{mrad})$ & 0.35 \\
\hline
\end{tabular}

in the transverse plane. To investigate resonance between the test particles and the mismatched core oscillation, we calculated the normalized test particle betatron (i.e., transverse) frequency and the normalized mismatched mode frequency as a function of current. The results are given in Fig. 1. Here, the transverse (lonitudinal) mode is a mode

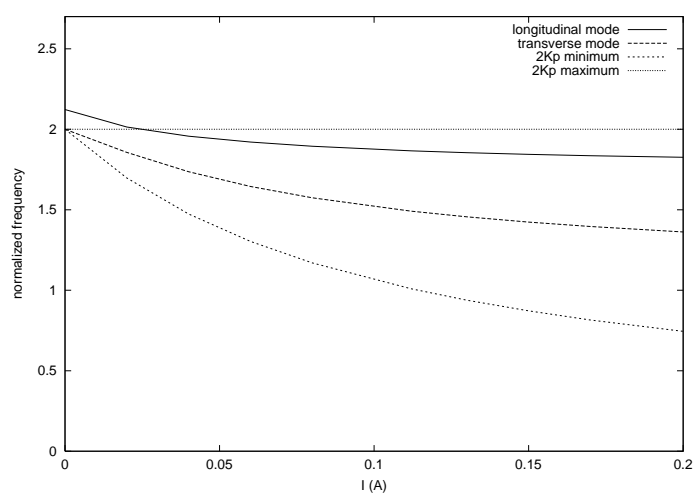

Figure 1: The test particle betatron frequency and core mode oscillation frequency as a function of current.

with a dominant transverse (longitudinal) oscillation. The test particle has a minimum frequency when it is inside the core and a maximum frequency when it is infinite far from the core. It is seen that there always exists a $2: 1$ resonance between the test particle betatron motion and the transverse 
core oscillation mode since the transverse mode frequency is within the range of twice of the particle frequency. This resonance is an "in-plane" resonance, since the test particle motion and the mode oscillation both involve the transverse plane. When the current is higher than some threshold value (that we call crossing point), there will also be a $2: 1$ resonance between the test particle and the longitudinal mode. We call this resonance a "cross-plane" resonance since it is between the transverse betatron motion of a test particle and the longitudinal core mode oscillation. Fig. 1 suggests that the cross-plane resonance can have a very large halo amplitude since the longitudinal mode frequency and twice the infinite amplitude test particle frequency are close. We also see that as the current decreases toward the crossing point current, the separation between the longitudinal mode frequency and twice the infinite amplitude test particle frequency decreases. This suggests that with decreasing current, the cross-plane resonance halo amplitude will become larger.

To see the resonance structure, we have made stroboscopic plots of the test particle betatron motion in the presence of only the longitudinal mode oscillation. Figure 2(a)
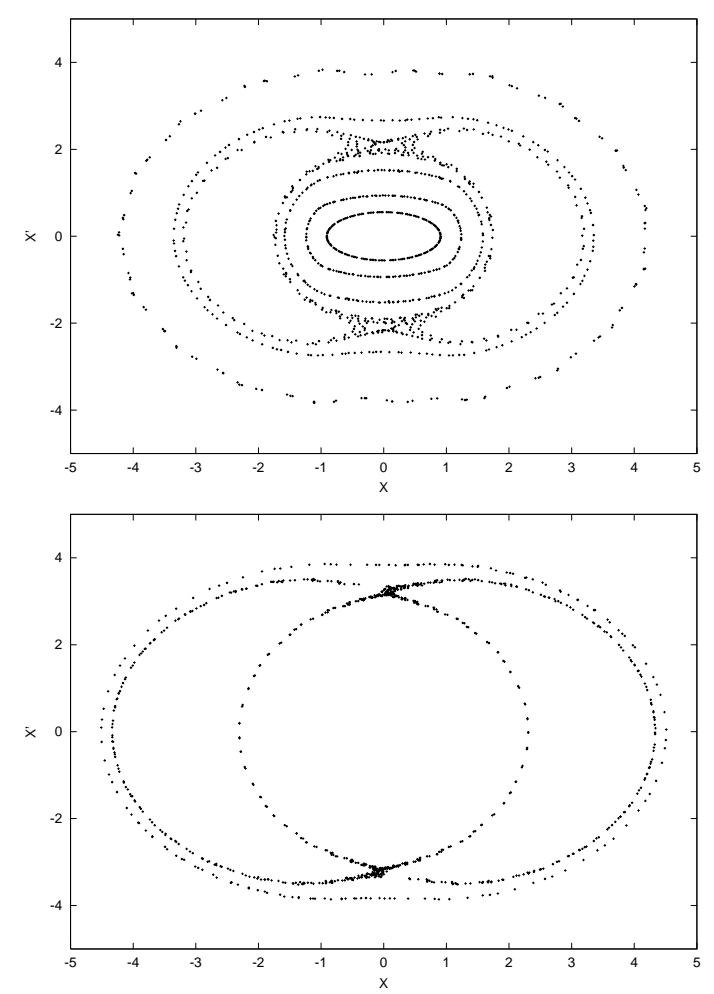

Figure 2: The stroboscopic plot in the $X-X^{\prime}$ plane at current (a) $112 \mathrm{~mA}$ and (b) $40 \mathrm{~mA}$ with a $10 \%$ initial transverse mismatch of longitudinal mode.

shows the stroboscopic plot in the transverse $X-X^{\prime}$ plane. The longitudinal mode was excited using a $10 \%$ transverse core mismatch and a $15 \%$ longitudinal mismatch at the design current of $112 \mathrm{~mA}$. We see that even with only $10 \%$ initial transverse mismatch, the halo amplitude is roughly three times the matched envelope size. Figure 2(b) shows similar transverse stroboscopic plots with $40 \mathrm{~mA}$ current. To excite the longitudinal mode, the initial transverse mismatch is still $10 \%$ and the longitudinal mismatch factor is $20 \%$. We see that the transverse halo extent increases from about three to 4.5 times the matched envelope size.

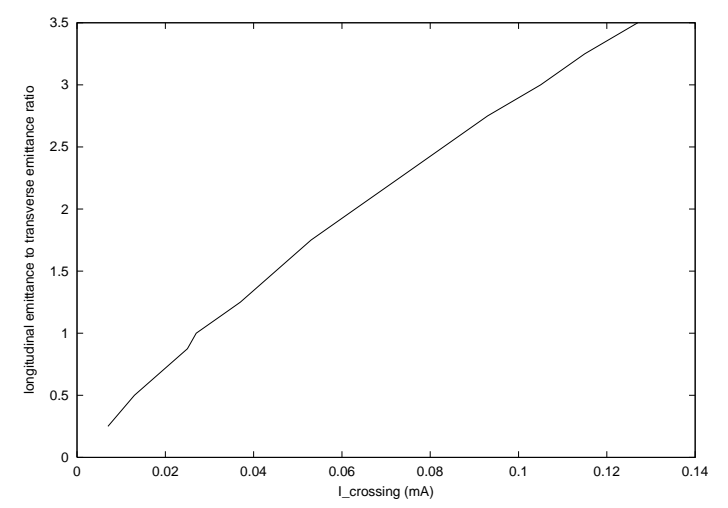

Figure 3: The longitudinal to transverse emittance ratio as a function of crossing-point current.

Next we studied the effect of varying emittance ratios and focusing strength ratios on the crossing point current. Figure 3 shows the emittance ratio as a function of crossing-point current, obtained by varying the longitudinal emittance with the other parameters fixed in Table 1 . Above the curve, there is no $2: 1$ cross-plane resonance. Below the curve, there exists a $2: 1$ cross-plane resonance. On the curve, the $2: 1$ resonance will occur with a test particle located at infinitely far distance, leading to infinitely large halo amplitude. As the emittance ratio increases, the crossing-point current increases. Fig. 4 shows the longitudinal-to-transverse focusing strength ratio as a function of crossing-point current, obtained by varying the longitudinal the longitudinal focusing strength with the other parameters fixed. It is seen that the cross-point cur-

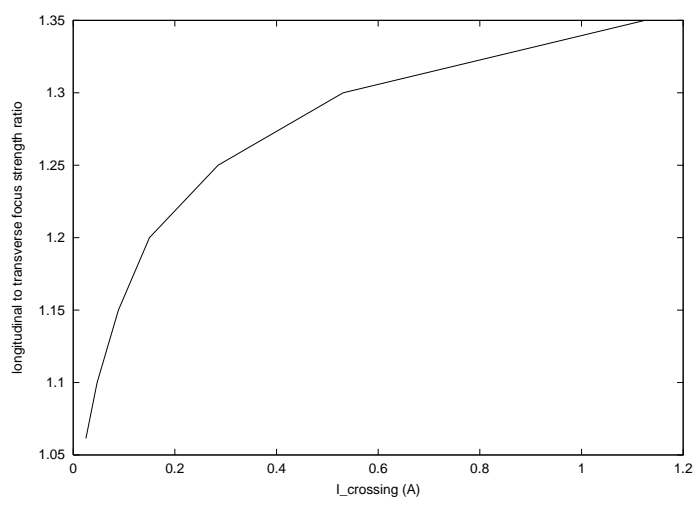

Figure 4: The longitudinal to transverse focusing strength ratio as a function of crossing-point current.

rent increases quickly with increasing focusing strength ratio. This suggests that the the focusing strength ratio would have a stronger effect on the cross-plane resonance than the emittance ratio. Since the halo will have an infinite trans- 
verse amplitude on the crossing-point line, the machine operation should stay away from this line in order to avoid the possibility of very large amplitude halo formation.

The cross-plane resonance could also happen between the longitudinal test particle and the transverse mode oscillation when the longitudinal-to-transverse focusing strength ratio is less than one. Fig. 5 shows the mode fre-

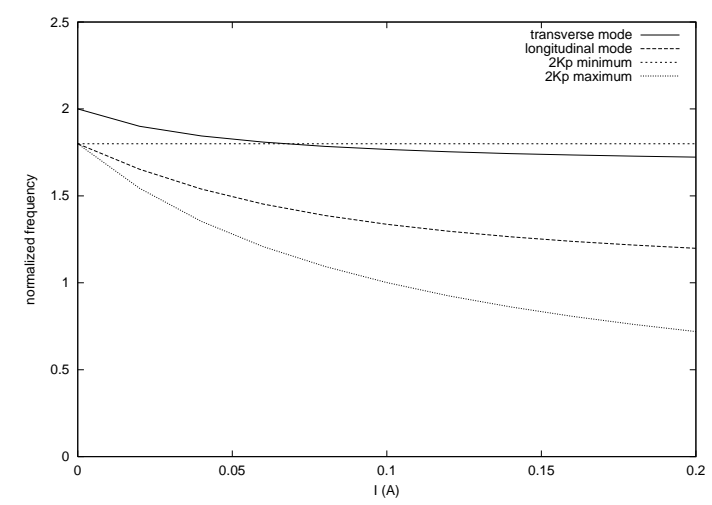

Figure 5: The test particle synchrotron frequency and core mode oscillation frequency as a function of current.

quency and the test particle synchrotron motion frequency as a function of current. Here, we have used the same parameters as in Table 1 except for a lower longitudinal focusing gradient, i.e., the longitudinal-to-transverse focusing strength ratio is 0.9. The nonlinear longitudinal RF field has been approximated by a linear focusing force. We see that there is a crossing-point around current $65 \mathrm{~mA}$ between the longitudinal test particle motion and the transverse mode oscillation. Fig. 6 shows the stroboscopic plot

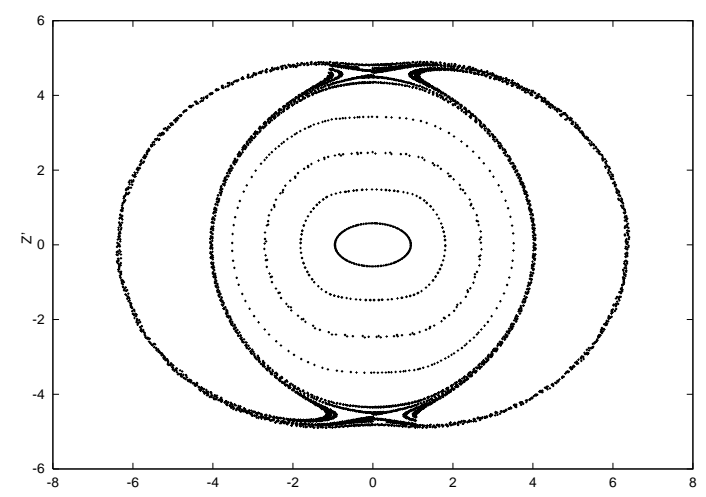

Figure 6: The stroboscopic plot of the particle synchrotron motion in the Z-Z' plane with linear RF field and initial $10 \%$ transverse mismatch of transverse mode.

of the test particle synchrotron motion in the longitudinal plane with initial $10 \%$ transverse mismatch and $5.6 \%$ longitudinal mismatch of the core transverse mode oscillation at a current of $80 \mathrm{~mA}$. It is seen that the longitudinal halo amplitude is six times the matched envelope size. This large amplitude halo could cause particles to move out of the RF bucket and be lost. However, in reality, the RF field is nonlinear. This nonlinear RF field will change the frequency spectrum of the particle synchrotron motion. The maximum synchrotron motion frequency is no longer that of the infinite distant test particle in the linear field approximation. Fig. 7 shows the stroboscopic plot of the test par-

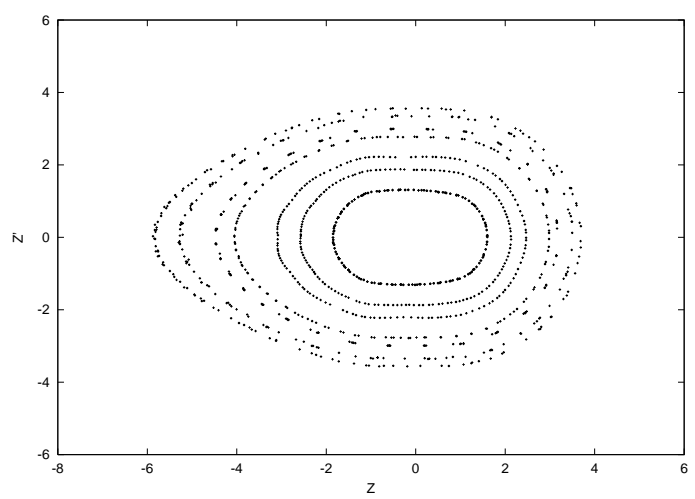

Figure 7: The stroboscopic plot of the particle synchrotron motion in the Z-Z' plane with nonlinear RF field and initial $10 \%$ transverse mismatch of transverse mode.

ticle synchrotron motion with RF nonlinearity. It is seen that there is no $2: 1$ resonance in the longitudinal plane.

\section{SUMMARY}

In this paper, we have shown that a very large-amplitude halo can be formed due to a cross-plane resonance. The halo amplitude increases as the current decreases toward the crossing point current. The longitudinal RF nonlinearity will help to prevent the very large-amplitude $2: 1$ resonance occuring. To avoid the large transverse amplitude halo, the machine operation should stay away from the crossing-point line in the emittance ratio and focusing strength ratio parameter space. This may have an important application in the high acceleration gradient superconducting linac design.

\section{REFERENCES}

[1] R. L. Gluckstern, Phys. Rev. Lett. 73, 1247 (1994).

[2] J. Lagniel, Nucl. Instrum. Methods Phys. Res. A 345, 46 (1994).

[3] R. D. Ryne and S. Habib, Part. Accl. 55, 365 (1996).

[4] R. L. Gluckstern, A. V. Fedotov, S. S. Kurennoy, and R. D. Ryne, Phys. Rev. E, 58, 4 (1998).

[5] T. P. Wangler, K. R. Crandall, R. Ryne, and T. S. Wang, Phys. Rev. ST Accel. Beams 1, 084201 (1998).

[6] M. Ikegami, Nucl. Instrum. Methods Phys. Res. A 435, 284 (1999).

[7] J. Qiang and R. Ryne,Phys. Rev. ST Accel. Beams 3, 064201 (2000).

[8] J. Stoval, et. al., "Superconducting Linac for the SNS," proceedings of XX International Linac Conference, Aug. 2125, 2000, Monterey, CA. 